\title{
Eficiencia, eficacia y transparencia del gasto público municipal
}

\author{
María Séfora Rodríguez Panduro \\ maria.sefora18@gmail.com \\ Gabriela del Pilar Palomino Alvarado \\ dpalominoal@ucvvirtual.edu.pe \\ Carlos Miguel Aguilar Saldaña \\ agua0122@hotmail.com \\ Escuela de post grado \\ Universidad César Vallejo
}

\section{RESUMEN}

La investigación está caracterizada esencialmente determinar la gestión del gasto público en el Municipio del Distrito de Juan Guerra. Presenta un estudio de investigación básica, con diseño no experimental, descriptivo de corte transversal. Como instrumento de recolección de datos se utilizó el cuestionario, en una muestra de 21 trabajadores entre nombrados y contratados. De esta manera se pudo realizar fehacientemente la valoración y aplicación de las normas; tanto a los trabajadores (16) y funcionarios (5). Por último, se logró establecer que existe un nivel bajo del gasto público desde la percepción del trabajador en un $87.5 \%$ y del funcionario en $80 \%$.

Palabras clave: Gasto, eficiencia, eficacia, trasparencia, presupuesto. 


\title{
Efficiency, effectiveness and transparency of municipal public spending.
}

\begin{abstract}
The investigation is essentially characterized to determine the management of public spending in the Municipality of the District of Juan Guerra. It presents a basic research study, with a non-experimental, descriptive cross-sectional design. The questionnaire was used as a data collection instrument, in a sample of 21 workers between appointed and hired. In this way, the assessment and application of the rules could be reliably carried out; both workers (16) and officials (5). Finally, it was established that there is a low level of public spending from the perception of the worker in $87.5 \%$ and the official in $80 \%$.
\end{abstract}

Keywords: Administration; finance, spending, internal control.

Artículo recibido: 25 oct. 2020 Aceptado para publicación: 07 nov. 2020 Correspondencia: maria.sefora18@gmail.com, Conflictos de Interés: Ninguna que declarar 


\section{INTRODUCCIÓN}

Existe una preocupación por poder mejorar el gasto público en la economía de Europa y hacer más rápida su proceso en la exportación y la industrialización. A pesar del crecimiento económico de la masa laboral. Por lo tanto, vemos que la inversión pública se acomoda con la inversión privada generando oportunidades en la industria, como la acertada capacidad para manejar el consumo público que realiza el Estado.

Los fondos públicos de los gobiernos regionales, departamentales, provinciales y distritales, vieron un decrecimiento al inicio y al término de su gestión estatal, la capacidad en las resoluciones de los gobernantes, crearon en la gestión una ruptura afectando a la ciudadanía e inversión privada deteniendo las reservas estatales. La Contraloría realizó una supervisión sobre el uso correcto del presupuesto público, detectando irregularidades en las unidades ejecutoras, lo que indica una carente administración pública (Gestión, 2018).

Asimismo, el Ministerio de Economía y Finanzas (MEF) en el año 2019, menciona en su informe anual del gasto del presupuesto por resultados, que la ejecución de inversión pública obtuvo un avance de $66 \%$ al cierre del 31 de diciembre, siendo los gobiernos locales quienes ejecutaron S/ 12,416 millones, un 14.8\% menos con respecto al 2018. Cabe señalar que entre las funciones con mayor gasto se encuentra transporte (61.9\%), seguido por saneamiento (57.2\%), educación (66.7\%) y agropecuaria $(65.5 \%)$.

La Contraloría pudo detectar hechos que generan inquietud en las finanzas públicas, el desaprovechamiento de los recursos que posee las instituciones estatales inspeccionadas. Hace hincapié en los desembolsos pendientes de pago, lo que genera descontrol en la entrada y salidas de bienes y/o servicios, siendo aprobada por los servidores públicos; lo que indica que son los mismos gobernantes quienes transgreden la norma, afectando la hacienda pública de los peruanos. La contraloría en su diligencia, asigna y prioriza que la Secretaría de Gestión Pública de la Presidencia de Consejo de Ministros prosiga a sancionar a los funcionarios públicos que realizan malversación de fondos públicos durante su mandato. (Diario Gestión, 2019).

Es importante establecer eficientemente y además efectivamente el gasto público, en las instituciones estatales, puesto que realizan gastos sobrevalorados, muchos estudios indican que si las instituciones se privatizaran los gastos públicos se disminuirían considerablemente y también se mejoraría la atención en las entidades estatales, no 
obstante, esa decisión política tiene que ser muy bien evaluada para que el país no se quede sin el control de los recursos mínimos para su progreso. (CEPAL, 2014).

\section{Gasto público}

Por el lado del gasto se considera que es productivo si entra como argumento en la función de producción y viceversa. Al respecto, Barro (1990) sostiene que, el gasto público genera la construcción de la economía del país, la cual fortalece el crecimiento de la producción complementado con la inversión privada fortaleciendo la base económica. Sus características se definen por las leyes presupuestales anuales, acorde con los planes de gobierno establecidos en las distintas instituciones del estado.

En el Perú, se vienen gestionando que el presupuesto asignado a las instituciones públicas genere resultados en beneficio de la ciudadanía, descrito en la política de modernización de la gestión pública (Ley $\mathrm{N}^{\circ}$ 27658). Considerada como la capacidad que tienen las instituciones públicas para hacer uso de los recursos económicos, recursos humanos y recursos físicos con pertinencia. Data de una extensa cadena histórica, empezando por los pensadores del país de China, Egipto, Grecia y Roma, luego se ve consolidado en el renacimiento del Continente de Europa y con ello la modernidad, en cuanto al uso de los recursos como una organización de institución de la sociedad, viniendo desde sus principios y creencias (Gómez \& De León, 2018).

Robinson \& Last (2015), indica que es un conjunto de acciones planificadas que permite destinar los presupuesto y recursos con los que se cuenta a través de las atenciones, con la finalidad de revertirlos a la sociedad de su entorno, teniendo en cuenta la medición que dará compromiso a los gestores de la institución, para brindar información y rendición de cuentas.

A través del MEF, (2018) se alude que el presupuesto por resultados (PPr), llevado a cabo según sus objetivos, establece resultados que se encuentre ligados a cambios para dar soluciones a los problemas que afecten a los ciudadanos generando compromisos y así lograrlos; identifica a través de un presupuesto, los requerimientos tanto en bienes como en servicio, lo necesario y que ello afecte el resultado; brindar información oportuna a la población sobre los gastos y las cuentas de la institución; cuidar y demostrar el correcto uso y funcionamiento de los recursos con los que cuenta la institución, durante el proceso de gestión; dar a conocer los logros obtenidos a favor del ciudadano, utilizar la información adquirida para fomentar el gasto futuro de la institución, facilitar a través 
de la rendición de cuentas anual y finalmente alcanzar la metas trazadas para obtener los incentivos que fortalezcan y mantengan el enfoque en los resultados (2018).

Asimismo, la importancia del PPR, contribuye en la mejora de gastos estatales, permitiendo que instituciones del estado tengan presente la efectividad de uso de los recursos con los que cuentan como instituciones, además de los productos tanto bienes y servicios que se esperan que generen el desarrollo y sobre todo mejore la condición de vida del ciudadano (Robinson \& Last, 2015). La toma de decisiones referente a la forma como se debe gestionar el gasto público, se debe ver influenciada por la información útil que brinda la ciudadanía respecto a sus necesidades prioritarias, como también a través de lo vertido por los trabajadores y autoridades, de ahí la importancia de que la planificación del presupuesto involucre a todos los actores de manera participativa. Acto democrático que se realizan con la finalidad de lograr la concertación y la participación ciudadana con el propósito de llegar a un consenso que permita elegir la opción que tenga mayor beneficio del ciudadano, son acciones en las cuales puede participar todo poblador y presentar sus propuestas acordes con las necesidades de su entorno (Robinson \& Last, 2015).

Por otro lado, en algunos casos la toma de decisiones se realiza de manera individual, referida a aquella que, por la condición del cargo, responsabilidad designada y las facultades entregadas por la institución los funcionarios lo realizan, lo cual debe estar definida en el manual y reglamento de organización y funciones (MOF y ROF) (Ataucusi, 2018). Práctica no recomendada, porque podría afectar directamente en el desempeño de la institución y no responder al bienestar de la ciudadanía.

La gestión del presupuesto por resultados nos permiten obtener las condiciones actuales en las cuales se encuentran la institución pública, generando la información oportuna para su fácil utilización la cual se encuentra determinada por los recursos financieros, que son aquellos recursos que se obtienen para los municipios y tienen connotaciones de ingresos corrientes, son asignados por el estado designado acorde con la planificación anual, ingresos que son recaudados por medio de los impuestos, también se podríamos decir del pago que hacen los ciudadanos la venta de los servicios, transferencias que son los aportes externos que bien de otros países y financiamiento son ingresos que se tuvo de anteriores periodos o a través de operaciones de crédito. (Morales, Barrera, Romero, Rodríguez, \& Távara, 2014). Asimismo, es el área de finanzas quien se encuentra como encargada de 
los recursos financieros y tiene como finalidad el manejo adecuado de dichos recursos, con un manejo mesurado y buscando la sostenibilidad de los limitados recursos que tiene la municipalidad.

Así también la captación de ingresos propios que, según Arias (2017), los impuestos que son cobrados en el área de rentas, tasas consideradas por porcentajes de acuerdo a los servicios brindados, multas y sanciones por las infracciones cometidas, venta de bienes que pertenecen a la institución, prestación de servicios lo que se realizan en beneficio de los pobladores y rentas de propiedad.

También tenemos las transferencias recibidas del Tesoro Público que son los ingresos que se capta a través de los presupuestos que designa el estado, con la finalidad de cumplir con la inversión pública que dinamice la economía y el cumplimiento de las necesidades de los ciudadanos (Ataucusi, 2018). Asimismo, los indicadores de evaluación presupuestal que, según Luna De Los Ríos (2016), menciona que se debe enfocar en medir la fehaciencia del recurso estatal, como se logró el presupuesto que fue aprobado para un periodo, no solo se trata de ver el grado de ejecución si no por el contrario se debe complementar con la calidad que genera beneficio para su población es decir la eficiencia y eficacia del mismo.

Es importante considerar que, la ciudadanía requiere conocer los procesos en los cuales se realiza la gestión de un gobierno a través de sus autoridades elegidas, así como de los trabajadores de la institución, el uso adecuado de los recursos, así como también el cumplimiento de los objetivos estratégicos; sin embargo, la medición del resultado solo se logra definir a través del cumplimiento de gasto público, pero no se logra el resultado para el cual fue invertido, por la forma de medirlo esta de manera errónea, es el ciudadano quien debe brindar la satisfacción de resultado obtenido (Robinson \& Last, 2015). En tal virtud, es importante caracterizar el gasto público en sus dimensiones siguientes:

\section{Eficiencia}

Es determinada por el uso adecuado de los recursos con los que cuenta un municipio, es decir, que la planificación se cumpla con los materiales y el tiempo establecido para generar el buen desempeño de cada gasto, la cual se debe medir y evaluar constantemente para realizar mejores estrategias o cambiar lo lineamientos de ser necesario (Robinson \& Last, 2015). 


\section{Eficacia}

Definida por la culminación de los objetivos institucionales planteados, siendo el uso de recursos proporcionales a las necesidades que se requieran para cumplirlo, tomando en cuenta el tiempo que se desea para lograrlo, se logra medir a través de los resultados obtenidos al finalizar un proyecto o un determinado tiempo (Robinson \& Last, 2015).

\section{Transparencia}

Definido por un mecanismo que evita el mal uso de los recursos públicos, el secreto, la improvisación, la ineficiencia, la discrecionalidad arbitraria y el abuso en el ejercicio de dicha función.

\section{MATERIALES Y MÉTODOS.}

El trabajo científico recurrió al método lógico deductivo, de manera tal, que se llevó a cabo la aplicación de los principios teóricos sobre la gestión del gasto público para analizar y determinar las caracterizaciones resaltantes de los nudos críticos de las dimensiones eficiencia, eficacia y transparencia en la municipalidad de Juan Guerra. La población conformada por 30 entre trabajadores y funcionarios y muestra de 21: 16 trabajadores y 5 funcionarios del área de administración, presupuesto, contabilidad, abastecimiento y rentas. Se excluyó a los que no laboraban en estas áreas. Se aplicó un cuestionario para caracterizar la eficiencia, eficacia y transparencia del gasto en la entidad, así el nivel de gestión del gasto público. Para el grado de confiabilidad se utilizó el Alfa de Cronbach, cuyo resultado de 0.8789 .

\section{RESULTADOS Y DISCUSIÓN}

El resultado arrojado de la investigación se obtuvo por la aplicación del cuestionario a los trabajadores y funcionarios de la municipalidad, y al mismo tiempo, se realizó el análisis e interpretación de la información obtenida. Se mantuvo de manera cerca los objetivos planteados.

Tabla 1. Nivel de la gestión del gasto público

\begin{tabular}{cccccccc}
\hline \multirow{2}{*}{ Nivel } & \multicolumn{2}{c}{ Escala } & \multicolumn{2}{c}{ Trabajadores } & \multicolumn{2}{c}{ Funcionarios } \\
& \multicolumn{2}{c}{ fi } & \% & fi \\
\hline Alto & 99 & - & 135 & 0 & $0.0 \%$ & 0 & $0.0 \%$ \\
Medio & $63-$ & 98 & 2 & $12.5 \%$ & 1 & $20.0 \%$ \\
Bajo & 27 & - & 62 & 14 & $87.5 \%$ & 4 & $80.0 \%$ \\
& Total & & $\mathbf{1 6}$ & $100.0 \%$ & $\mathbf{5}$ & $100.0 \%$ \\
\hline
\end{tabular}

Fuente: cuestionario aplicado a trabajadores y funcionarios de la municipalidad distrital de Juan Guerra 
La percepción del gasto público según la percepción de los trabajadores de la municipalidad de Juan Guerra es "Bajo" en un 87.5\% (14) y "Medio" en un 12.5\% (2).

El resultado es similar, según la percepción de los funcionarios; el 80\% (4) lo ubica en el nivel "Bajo" y el 20\% (1) en "Medio" de acuerdo a nuestra escala de medición.

Tabla. 2. Eficiencia en el gasto público desde la perspectiva de los trabajadores.

\begin{tabular}{|c|c|c|c|c|c|c|c|}
\hline & \multirow[t]{2}{*}{ Eficiencia } & \multicolumn{2}{|c|}{$\begin{array}{c}\text { Nunca/ casi } \\
\text { nunca }\end{array}$} & \multicolumn{2}{|c|}{$\begin{array}{l}\text { Algunas } \\
\text { veces }\end{array}$} & \multicolumn{2}{|c|}{$\begin{array}{l}\text { Siempre/ casi } \\
\text { siempre }\end{array}$} \\
\hline & & fi & $\%$ & fi & $\%$ & fi & $\%$ \\
\hline \multirow{6}{*}{$\begin{array}{l}\text { Desempeño } \\
\text { Personal }\end{array}$} & Cuenta con perfil de puesto en el área & 12 & $75.0 \%$ & 4 & $25.0 \%$ & 0 & $0.0 \%$ \\
\hline & Cumplimiento de objetivos institucionales & 12 & $75.0 \%$ & 4 & $25.0 \%$ & 0 & $0.0 \%$ \\
\hline & Planificación del presupuesto en base a objetivos & 12 & $75.0 \%$ & 4 & $25.0 \%$ & 0 & $0.0 \%$ \\
\hline & $\begin{array}{l}\text { Evaluaciones de resultados de gasto en el logro } \\
\text { de sus metas }\end{array}$ & 15 & $93.8 \%$ & 1 & $6.3 \%$ & 0 & $0.0 \%$ \\
\hline & Control de gasto según área de trabajo & 12 & $75.0 \%$ & 4 & $25.0 \%$ & 0 & $0.0 \%$ \\
\hline & $\begin{array}{l}\text { Información oportuna de compromiso, } \\
\text { devengado y pagado }\end{array}$ & 6 & $37.5 \%$ & 7 & $43.8 \%$ & 3 & $18.8 \%$ \\
\hline \multirow{4}{*}{ Recursos } & El área de trabajo cuenta con el personal mínimo & 13 & $81.3 \%$ & 3 & $18.8 \%$ & 0 & $0.0 \%$ \\
\hline & Uso de recursos oportuna, confiable y útil & 12 & $75.0 \%$ & 4 & $25.0 \%$ & 0 & $0.0 \%$ \\
\hline & $\begin{array}{l}\text { Control y supervisión permanente sobre los } \\
\text { recursos que maneja }\end{array}$ & 7 & $43.8 \%$ & 8 & $50.0 \%$ & 1 & $6.3 \%$ \\
\hline & $\begin{array}{l}\text { actualización del SIAF y SIGA en su equipo } \\
\text { tecnológico }\end{array}$ & 7 & $43.8 \%$ & 8 & $50.0 \%$ & 1 & $6.3 \%$ \\
\hline
\end{tabular}

Fuente: Cuestionario aplicado a los trabajadores de la municipalidad de Juan Guerra

\section{Interpretación}

Del 100\% (16) de los trabajadores, referente al desempeño laboral, el 93.8\% (15) indicaron "Nunca o casi nunca" realizan evaluaciones de resultados de gasto en el logro de sus metas; ni cuenta con el perfil de puesto en el área, ni cumple los objetivos institucionales, ni planifica el presupuesto en base a los objetivos, ni control de gasto según área de trabajo 75\% (12).

Finalmente, referente a los recursos, el $81.3 \%$ (13) de los colaboradores manifiestan que "Nunca o casi nunca" cuenta con el personal mínimo necesario en el área, ni utiliza los recursos de manera oportuna, confiable y útil en un 75\% (12). Así mismo, el 50\% (8) considera que "Algunas veces" existe un control de los recursos y se actualiza los equipos tecnológicos con el Sistema Integrado de Administración Financiera (SIAF) y el Sistema Integrado de Gestión Administrativa (SIGA). 
Tabla. 3. Eficacia en el gasto público desde la perspectiva de los trabajadores.

\begin{tabular}{|c|c|c|c|c|c|c|c|}
\hline & \multirow{2}{*}{ Eficacia } & \multicolumn{2}{|c|}{$\begin{array}{c}\text { Nunca/ casi } \\
\text { nunca }\end{array}$} & \multicolumn{2}{|c|}{$\begin{array}{l}\text { Algunas } \\
\text { veces }\end{array}$} & \multicolumn{2}{|c|}{$\begin{array}{l}\text { Siempre/ casi } \\
\text { siempre }\end{array}$} \\
\hline & & fi & $\%$ & fi & $\%$ & fi & $\%$ \\
\hline \multirow{3}{*}{ Gasto } & $\begin{array}{l}\text { Ejecución del gasto de acuerdo a lo } \\
\text { programado presupuestalmente }\end{array}$ & 4 & $25.0 \%$ & 7 & $43.8 \%$ & 5 & $31.3 \%$ \\
\hline & $\begin{array}{l}\text { Ejecución de los procedimientos del } \\
\text { devengado de acuerdo a las normativas de la } \\
\text { DNTP }\end{array}$ & 3 & $18.8 \%$ & 10 & $62.5 \%$ & 3 & $18.8 \%$ \\
\hline & $\begin{array}{l}\text { Sustentación con documentos requeridos } \\
\text { para los compromisos de gastos }\end{array}$ & 4 & $25.0 \%$ & 11 & $68.8 \%$ & 1 & $6.3 \%$ \\
\hline \multirow{2}{*}{ Cumplimiento } & $\begin{array}{l}\text { Realización de gastos de bienes y servicios } \\
\text { de acuerdo al Plan Anual de Adquisiciones }\end{array}$ & 6 & $37.5 \%$ & 9 & $56.3 \%$ & 1 & $6.3 \%$ \\
\hline & $\begin{array}{l}\text { Cumplimiento de metas establecidas de } \\
\text { acuerdo a lo programado }\end{array}$ & 4 & $25.0 \%$ & 10 & $62.5 \%$ & 2 & $12.5 \%$ \\
\hline Evaluar & Evaluación presupuestal semestral y anual & 12 & $75.0 \%$ & 4 & $25.0 \%$ & 0 & $0.0 \%$ \\
\hline
\end{tabular}

Fuente: Cuestionario aplicado a los trabajadores de la municipalidad de Juan Guerra

\section{Interpretación}

Los resultados arrojados de la investigación sobre la dimensión eficacia del gasto público en la municipalidad, en relación al gasto, el 68\% (11) refiere que "Algunas veces" se sustentan con los documentos requeridos para el gasto público, ni se ejecuta los procedimientos del devengado de acuerdo a las normas de la Dirección Nacional de Tesorería Pública, el 62.5\% (10).

Asimismo, respecto al cumplimiento, el 62\% (10) refiere que "Algunas veces" se cumple las metas establecidas de acuerdo a lo programado, ni se realiza los gastos de bienes y servicios de acuerdo al Plan Anual de Contrataciones en un 56.3\% (9).

Por último, referente a la evaluación, 75\% (12) considera que "Nunca o casi nunca" se realiza la evaluación presupuestal en forma semestral y anual del gasto público.

Tabla. 4. Transparencia en el gasto público desde la perspectiva de los trabajadores.

\begin{tabular}{|c|c|c|c|c|c|c|c|}
\hline & \multirow[t]{2}{*}{ Transparencia } & \multicolumn{2}{|c|}{$\begin{array}{l}\text { Nunca/ casi } \\
\text { nunca }\end{array}$} & \multicolumn{2}{|c|}{$\begin{array}{l}\text { Algunas } \\
\text { veces }\end{array}$} & \multicolumn{2}{|c|}{$\begin{array}{l}\text { Siempre/ casi } \\
\text { siempre }\end{array}$} \\
\hline & & fi & $\%$ & fi & $\%$ & fi & $\%$ \\
\hline \multirow{2}{*}{ Gestión } & $\begin{array}{l}\text { Conocimiento a la comunidad de asignación } \\
\text { de mayores recursos }\end{array}$ & 12 & $75.0 \%$ & 4 & $25.0 \%$ & 0 & $0.0 \%$ \\
\hline & $\begin{array}{l}\text { Participas o haz participado en la elaboración } \\
\text { del presupuesto anual }\end{array}$ & 16 & $100.0 \%$ & 0 & $0.0 \%$ & 0 & $0.0 \%$ \\
\hline Contrataciones & $\begin{array}{l}\text { Conocimiento de licitaciones públicas en la } \\
\text { contratación de bienes y obras }\end{array}$ & 13 & $81.3 \%$ & 3 & $18.8 \%$ & 0 & $0.0 \%$ \\
\hline
\end{tabular}


Difusión en el portal Institucional las contrataciones de Bienes servicios, obras y se publican oportunamente en el SEACE

Actualización del portal de trasparencia y el

Evaluaciones nivel de avance de ejecución.

$\begin{array}{llllll}5 & 31.3 \% & 9 & 56.3 \% & 2 & 12.5 \% \\ 15 & 93.8 \% & 1 & 6.3 \% & 0 & 0.0 \% \\ 16 & 100.0 \% & 0 & 0.0 \% & 0 & 0.0 \%\end{array}$

Publicación de reportes de evaluaciones en forma semestral y anual

Fuente: Cuestionario aplicado a los trabajadores de la municipalidad de Juan Guerra

\section{Interpretación}

Referente a la gestión, 100\% (16) considera que "Nunca o casi nunca" participa o ha participado en la elaboración del presupuesto anual, tampoco se da a conocer a la comunidad la asignación de mayores recursos en un 75\% (12).

Concerniente a las contrataciones, el $81.3 \%$ (13) manifiesta que "Nunca o casi nunca" conoce si en la entidad realiza licitaciones públicas en la contratación de bienes y obras mayores a 8 UIT, así mismo un 56.3\% (9) “Algunas veces" se difunden oportunamente en el Sistema Electrónico de Contrataciones del Estado (SEACE) y en el portal Institucional las contrataciones de bienes servicios y obras.

Asimismo, en relación a las evaluaciones, el 100\% (16) refiere que "Nunca o casi nunca" se realiza las publicaciones de los reportes de evaluaciones en forma semestral y anual del gasto público; de igual forma 93.8\% (15) considera que no se gestiona la actualización del portal de trasparencia en el nivel de avance de ejecución del gasto público.

\section{Eficiencia del gasto público desde la perspectiva del funcionario}

Respecto al desempeño personal, 100\% (5) manifiesta que "Nunca o casi nunca" se desarrolla el control de gasto por parte de los funcionarios; ni se gestiona el cumplimiento de los objetivos institucionales; asimismo, no se aprueba ni se recepciona oportunamente dentro los plazos establecidos los trabajos del gasto público de parte de los colaboradores en un $80 \%$ (4). De igual forma no se evalúa los resultados de gasto en el logro de las metas. "Algunas veces" el funcionario cumple con el perfil de puesto según el área que desempeña, el $80 \%$ (4).

En relación a las normas, el 100\% (5) considera que "Nunca o casi nunca" se planifica la gestión del gasto público de acuerdo a los documentos normativos ni se ejecuta de acuerdo a las normas legales establecidos por la Dirección Nacional de Presupuesto Público. El 80\% (4) refiere que “Algunas veces” se tiene en cuenta los plazos establecidos 
para la presentación del presupuesto al MEF, ni se monitorea la ejecución del gasto público, según los documentos normativos, el 60\% (3).

Por último, referente a los recursos, el 80\% (4) considera que "Nunca o casi nunca" se realiza de manera programada el control del uso de recursos ni se planifica el soporte técnico permanentemente del sistema SIAF y SIGA en los equipos tecnológicos. El 60\% (3) refiere que "Algunas veces" se gestiona que las áreas de trabajo cuenten con el personal mínimo necesario y se considera que los recursos utilizados por los colaboradores son oportuna, confiable y útiles

\section{Eficacia del gasto público desde la perspectiva del funcionario}

Respecto al gasto, el $80 \%$ (4) de los funcionarios refiere que "Nunca o casi nunca" se vigila de manera permanente la ejecución del gasto de acuerdo a lo programado en el presupuesto. El 60\% (3) considera que "Algunas veces" se tiene conocimiento de los compromisos adquiridos con los acreedores.

Referente al cumplimiento, el $80 \%$ (4) manifiesta que "Nunca o casi nunca" se realizan acciones de coordinación entre funcionarios para la ejecución del gasto y cumplimiento de las metas establecidas; el 60\% (3) manifiesta que "Algunas veces" se realiza acciones de cumplimiento del Plan Anual de Adquisiciones.

Por último, concerniente a la evaluación, el 60\% (3) expresa que "Nunca o casi nunca" se supervisa el cumplimiento de la evaluación presupuestal en forma semestral y anual.

\section{Transparencia del gasto público desde la perspectiva del funcionario}

Concerniente a la gestión, el 100\% (5) refiere que "Nunca o casi nunca" se comunica a la comunidad sobre la asignación de mayores recursos. De igual forma considera que "Algunas veces" se planifica el presupuesto anual de manera conjunta con todos los actores.

En relación a las contrataciones, el 100\% (5) manifiesta que "Nunca o casi nunca" se ejecutan de acuerdo a la Ley de contrataciones para la adquisición de bienes y obras. El $80 \%$ (4) considera que "Algunas veces" se publican oportunamente en el SEACE y se difunde en el portal institucional sobre las contrataciones de bienes servicios y obras.

Referente a la evaluación, el $80 \%$ (4) considera que "Nunca o casi nunca" se gestiona la actualiza el portal de transparencia y se publica el nivel de avance de ejecución del gasto público, ni se analiza los resultados en forma semestral y anual para su publicación de los reportes de evaluaciones, el $60 \%$ (3). 


\section{DISCUSIÓN}

La investigación analiza y discute los resultados reflejados sobre la gestión del Gasto Público en la municipalidad del distrito de Juan Guerra, 2020. Teniendo en cuenta el primer objetivo específico de determinar el nivel de gestión del gasto público, se observa, que existe una percepción de nivel "Bajo" por parte de los trabajadores en un 87.5\%, y $80 \%$ por parte de los funcionarios de la municipalidad sobre el gasto público.

En una institución es importante tener en cuenta la calidad del uso de los recursos en base a las normas o leyes estipuladas en el país, para el cumplimiento de manera loable del gasto público municipal. Todo esto conduce al cumplimiento de los objetivos y metas propuestas por la entidad.

Teniendo en cuenta el segundo objetivo, podemos resaltar que la eficiencia del gasto público es deficiente ya que los trabajadores no cuentan con perfil de puesto, ni cumplen los objetivos institucionales, ni se planifica el presupuesto, ni se realiza evaluaciones del gasto. De igual forma no existe un control del gasto, ni personal mínimo requerido por las áreas, ni adecuado uso de los recursos. Por otra parte, lo ineficaz que es el gasto se establece a través de que no se realiza evaluación presupuestal semestral y anual. Asimismo, la dimensión de transparencia demuestra que es poco transparente referente a la información que brinda a la comunidad a través del portal web institucional, aún más con la participación de los responsables del personal de las áreas comprometidas con el gasto.

Es importante que toda institución municipal realice sus acciones futuras a través de la planificación estratégica teniendo en cuenta el personal idóneo con conocimiento de la gestión pública, una buena planificación de los gastos, que estos se realicen un control permanente sobre lo ejecutado. Estas acciones brindarán soportes para la administración. Una comunidad informada y bien comunicada, hace que se reduzcan los conflictos sociales.

La información reflejada en base al tercer objetivo específico, se evidencia la aplicabilidad de procesos para medir los resultados, debido a ello que la planificación, el presupuesto por resultados gestión de recursos financieros y monitoreo de evolución son inadecuados en la municipalidad.

Máttar, (2014), refiere que los distintos procesos que desconocen y la falta de conocimiento técnico de las personas que trabajan y más aún de las autoridades que son 
electas, trae consigo una serie de irregularidades en su mala aplicación como la malversación de fondos, la corrupción, la colusión, y el tráfico de influencias, para hacer el uso de los recursos de las instituciones sin generar el control y los resultados para las cuales fueron creadas.

Es necesario que la entidad contrate y capacite al personal sobre el gasto público, se realice un adecuado control por procesos para medir los resultados, se establezcan manuales de procesos para cumplir con eficacia, eficiencia y transparencia del gasto público; de esa manera se reduce problemas legales futuros e incumplimiento de las metas y objetivos en la municipalidad.

En tal sentido, frente a la información recabada podemos determinar que existe dificultades y limitaciones en la gestión del presupuesto por resultados lo que da nacimiento a la necesidad de implementar un apropiado presupuesto por resultados que permita la estricta aplicación de las normas con un adecuado Plan Estratégico y cumplimiento de los objetivos trazados; de esta forma controlar acciones importantes y mejorar el gasto público. Jiang \& Gong, (2019), concluye que, el presupuesto es la base de la gestión del desempeño del presupuesto, por lo tanto, un plan presupuestario efectivo es importante para el sistema fiscal.

\section{CONCLUSIONES}

En conclusión, podemos manifestar que:

1. El $87.5 \%$ de los trabajadores y $80 \%$ de los funcionarios percibe que el nivel de gasto público es "Bajo", debido a que no es eficiente, eficaz y transparente su ejecución.

2. Desde la perspectiva del trabajador la institución, se caracteriza prioritariamente por que "Nunca o casi nunca" se cuenta con el perfil de puesto en el área, se cumple los objetivos institucionales, la planificación del presupuesto en base a objetivos, control del gasto según área de trabajo, ejecución de todo lo planificado, contar con personal mínimo necesario según área, uso de recursos de manera oportuna, confiable y útil.

3. Desde la perspectiva del funcionario, el gasto público se caracteriza porque "Nunca o casi nunca", se gestiona el cumplimiento de los objetivos institucionales de manera eficiente, aprobación y recepción de los informes de trabajo en los plazos establecidos, evaluación de los resultados para el logro de las metas, planificación del presupuesto de acuerdo a los documentos normativos. Por último, no se realiza de manera programada el control del uso de recursos ni se planifica el soporte técnico permanentemente del sistema SIAF y SIGA en los equipos tecnológicos. 
4. La entidad tiene la imperiosa necesidad de plasmar estrategias de compromiso de participación de los trabajadores de las áreas comprometidas con el gasto público en la elaboración o actualización del Plan Estratégico, Plan Operativo institucional y Programa de Compromiso Anuales. Es necesario recurrir a diplomacias de comunicación asertiva para un adecuado flujo de información interna sobre el techo presupuestal de la institución y ejecución de presupuesto en Programas Presupuestales. Asimismo, urge la necesidad de establecer el seguimiento trimestral y de un manual de procesos que conduzcan al cumplimiento de los plazos definidos sobre la ejecución eficiente de la gestión financiera en los programas presupuestales, teniendo en cuenta la gestión financiera y su relación con las metas establecidas en el Plan Operativo Institucional anual.

\section{LISTA DE REFERENCIA}

Abusasa, R., Cusato, A., \& Pastor, C. (2008). Eficiencia del gasto en el Perú. Instituto Peruano de Economía.

Alegría, D. (2017). Human resources capacities in good practices of the financial administration system in the provincial municipality of Leoncio Prado. Huánuco Region 2016. (Scientific article), Peru. Recovered from http://revistas.unas.edu.pe/index.php/Balances/article/view/112.

Alfageme, M., \& Guabloche, M. (s.f.). Estado, gasto público y desarrollo de las capacidades. Estudios económicos.

Amat, Soldevilla, \& Castelló. (2006). Planificación presupuestaria.

Arias, D. (2014). Control interno en la ejecución presupuestal del gasto público en la municipalidad distrital de Llacanora años 2012-2013. Cajamarca.

Barcelata, H. (2019) Deuda pública subnacional y desarrollo económico local. (Artículo científico). Universidad Autónoma Metropolitana, España. Recuperada de http://www.scielo.org.mx/scielo.php?script=sci_abstract\&pid=S0188$33802019000200165 \& \operatorname{lng}=$ es\&nrm=iso.

Bastidas, C., Andocilla, J., \& Franco, W. (2016). Consideraciones sobre calidad de gasto público.

Cabrera, C., Fuentes, Cerezo, G. La gestión financiera aplicada a las organizaciones. (Artículo Científico)Universidad Técnica Estatal de Quevedo. EcuadorRecuperada de http://dominiodelasciencias.com/ojs/index.php/es/index. 
Campos, C. (2003). Los procesos de control interno en el departamento de ejecución presupuestal de una institución del estado. Lima - Perú.

CEPLAN. (s.f.). Planeamiento estratégico en las instituciones públicas.

Colina, L., \& Cubillan, A. (2012). La planificación presupuestaria en universidades públicas. Centro de investigación de ciencias administrativas y gerenciales.

Constitución Política del Perú. (1993). Perú.

Elorrega, G. (2002). Sistema de control interno.

Escobar, D., \& Hermoza, G. (2015). El presupuesto por resultados en la calidad del gasto público de la unidad ejecutora de la dirección regional de salud Huancavelica año 2014. Huancavelica-Perú.

Gómez Meneses, F. E., \& Zarate Camelo, M. A. (2011). Gasto público en educación frente al comportamiento de los principales agregados económicos en Latinoamérica. Finanzas y política económica.

Ley 28411. (2004). Ley General del Sistema Nacional de Presupuesto. Perú. Recuperado de

https://cdn.www.gob.pe/uploads/document/file/255721/229463_file2018121816260-1twi55b.pdf.

López Casasnoovas, G., \& Castellanos, A. (2003). La calidad del gasto y la mejora de la eficiencia en el sector público. una valoración del gasto funcional desde las Aa.Pp. desde una perspectiva multijurisdiccional. Dialnet.

Olivos Campos, C., \& Quiñones Jaico, V. D. (2016). Análisis de la eficiencia del gasto público de la municipalidad provincial de Chiclayo y su impacto socio económico. Ciencia y tecnología.

Paco Matamoros, L., \& Mantari Araujo, W. S. (2014). El proceso de ejecución presupuestal y su influencia en la calidad de gasto de la municipalidad provincial de Huancavelica periodo 2013. Perú.

Paiva, F. (2013). Internal control and its impact on the profitability of the company cobros del norte S.A. (Scientific Article) UNAN-Managua. FAREM Nicaragua. Recovered from https://repositorio.unan.edu.ni/5910/3/99-371-1-PB.pdf 
Prieto Hermoza, M. I. (2012). Influencia de la gestión del presupuesto por resultados en la calidad de gasto en las municipalidades del Perú 20062-010 Caso: lima, Junín y Ancash. Lima-Perú.

Resolución de Secretaría General N R.S.G 045-2020-CG. 08 de abril, 2020. Recuperada de https://www.gob.pe/institucion/contraloria/normas-legales/606112-r-s-g-0452020-cg-sge.

Resolución de Contraloría. N 100-2020-CG. Control Simultáneo. 28 de marzo, 2020. Diario Oficial el peruano. https://busquedas.elperuano.pe/normaslegales/adicionan-sexta-disposicioncomplementaria-final-a-la-direct-resolucion-n-100-2020-cg-1865210-1/

Recovered from https://repositorio.espe.edu.ec/bitstream/21000/10019/1/AC-ESPELCAI-0431A.pdf

Terrazas, R. (2009) Modelo de gestión financiera para una organización. (Artículo Científico) Universidad Católica Boliviana San Pablo. Bolivia. Recuperada de https://www.redalyc.org/pdf/4259/425942159005.pdf

Vargas, S. (2018). Sistemas administrativos y gestión por resultados en la Municipalidad Provincial de San Martín- 2018. Perú (Tesis de Doctorado) Universidad César vallejo.

Recuperado-de https://alicia.concytec.gob.pe/vufind/Record/UCVV_0b449707635283c6f9e421 da6364333b/Details. 